\title{
Death-associated protein kinase 1 mediates $A \beta 42$ aggregation-induced neuronal apoptosis and tau dysregulation in Alzheimer's disease
}

\author{
Tao Zhang\#, Yongfang Xia\#, Li Hu, Dongmei Chen, Chen-Ling Gan, Long Wang, Yingxue Mei, Guihua \\ Lan, Xindong Shui, Yuan Tian, Ruomeng Li, Mi Zhang, Tae Ho Lee ${ }^{\bowtie}$ \\ Fujian Key Laboratory of Translational Research in Cancer and Neurodegenerative Diseases, Institute for Translational Medicine, School of Basic Medical \\ Sciences, Fujian Medical University, Fuzhou, Fujian 350122, China. \\ \# These authors contributed equally to this work. \\ $\triangle$ Corresponding author: Tae Ho Lee, Fujian Medical University, 1 Xuefu North Road, Fuzhou, Fujian 350122, China; E-mail: tlee0813@fjmu.edu.cn; \\ leethres@hotmail.com; Tel.: +86-591-2286-2498; Fax: +86-591-2286-2320. \\ (C) The author(s). This is an open access article distributed under the terms of the Creative Commons Attribution License (https://creativecommons.org/licenses/by/4.0/). \\ See http://ivyspring.com/terms for full terms and conditions.
}

Received: 2021.09.02; Accepted: 2021.11.27; Published: 2022.01.01

\begin{abstract}
The aggregation of amyloid- $\beta$ (A $\beta$ ) peptides into oligomers and fibrils is a key pathological feature of Alzheimer's disease (AD). An increasing amount of evidence suggests that oligomeric $A \beta$ might be the major culprit responsible for various neuropathological changes in AD. Death-associated protein kinase 1 (DAPK 1 ) is abnormally elevated in brains of $A D$ patients and plays an important role in modulating tau homeostasis by regulating prolyl isomerase Pin 1 phosphorylation. However, it remains elusive whether and how $A \beta$ species influence the function of DAPK 1 , and whether this may further affect the function and phosphorylation of tau in neurons. Herein, we demonstrated that $A \beta$ aggregates (both oligomers and fibrils) prepared from synthetic $A \beta 42$ peptides were able to upregulate DAPK1 protein levels and thereby its function through heat shock protein 90 (HSP90)-mediated protein stabilization. DAPK1 activation not only caused neuronal apoptosis, but also phosphorylated Pin 1 at the Ser71 residue, leading to tau accumulation and phosphorylation at multiple AD-related sites in primary neurons. Both DAPK 1 knockout $(\mathrm{KO})$ and the application of a specific DAPK1 inhibitor could effectively protect primary neurons against $A \beta$ aggregate-induced cell death and tau dysregulation, corroborating the critical role of $D A P K 1$ in mediating $A \beta$ aggregation-induced neuronal damage. Our study suggests a mechanistic link between $A \beta$ oligomerization and tau hyperphosphorylation mediated by DAPK 1, and supports the role of DAPK 1 as a promising target for early intervention in $A D$.
\end{abstract}

Key words: Amyloid- $\beta$ (A $\beta$ ), Death-associated protein kinase 1 (DAPK1), Phosphorylation, Tau, Oligomer

\section{Introduction}

Alzheimer's disease (AD) is the leading cause of dementia, accounting for more than $60 \%$ of total dementia cases worldwide [1]. Pathologically, AD is characterized by the deposition of amyloid- $\beta$ (A $\beta$ ) peptides in the brain parenchyma and the accumulation of hyperphosphorylated tau proteins in the form of neurofibrillary tangles in neurons [1]. Clinical evidence has suggested that the accumulation of $A \beta$ in the brain is the earliest detectable event in the progression of $\mathrm{AD}$, occurring about a decade prior to the onset of cognitive dysfunction [2]. Similar to $A \beta$ accumulation in the parenchyma, the content of tau proteins has also been found to increase in cerebrospinal fluid (CSF) about 10 years before symptom onset [2]. The early initiation of both $A \beta$ and tau accumulation in the disease course of $\mathrm{AD}$ highlights the importance of these two proteins as targets for disease diagnosis and intervention.

$A \beta$ oligomers are important intermediates formed during the self-assembly of $A \beta$ and cover a wide range of molecular weights and morphologies [3]. For example, Lesné et al. found that $A \beta$ dimers, 
trimers and $\mathrm{A} \beta * 56(\sim 56 \mathrm{kDa})$ are present in human brains, and the contents of these oligomers increase gradually with age [4]. These types of oligomers are small in size and are therefore regarded as low molecular weight (LMW) species. Yang et al. identified a different group of soluble $A \beta$ aggregates with high molecular weight (HMW) from the brains of AD patients. These species range from $\sim 150$ to 600 $\mathrm{kDa}$ based on size exclusion chromatography analysis [5]. Although $A \beta$ oligomers remain low in abundance and metastable in the CSF, they are usually soluble and diffusible in solution and manifest high structural heterogeneity, making these species rather detrimental to neurons $[3,6]$. A $\beta$ oligomers have been proven to trigger tau phosphorylation in neurons, resulting in microtubule dysfunction and neurite degeneration. For instance, $A \beta$ oligomers may activate protein kinases such as glycogen synthase kinase-3 $\beta$ (GSK-3 $\beta), \quad \mathrm{Ca}^{2+}$-dependent calmodulin kinase IIa (CaMKIIa) and cyclin-dependent kinase 5 (CDK5) that are able to directly phosphorylate tau proteins at AD-related sites [7-9]. The exacerbation of tau pathology by $A \beta$ oligomers implicates a toxic interplay between these two pathological changes in the central nervous system (CNS).

Death-associated protein kinase 1 (DAPK1) is a $\mathrm{Ca}^{2+}$ /calmodulin-dependent serine/threonine kinase. DAPK1 plays a key role in modulating cell death and autophagy and is intimately involved in the pathogenesis of tumors, ischemic brain injury and neurodegenerative diseases [10, 11]. Several studies have suggested that DAPK1 might be a risk factor for sporadic AD. For example, our previous study revealed for the first time that the DAPK1 protein level in the hippocampus of AD cases is significantly higher than that of controls [12]. DAPK1 upregulation in AD not only affects $A \beta$ pathology by altering APP phosphorylation and processing, but also influences the function of tau through multiple pathways [13]. DAPK1 activation phosphorylates Pin1 at the Ser71 residue and inactivates its isomerization capability, which stabilizes tau proteins and promotes tau phosphorylation in the brain [12, 14]. In addition, DAPK1 may indirectly induce tau phosphorylation at the Ser262 residue via microtubule affinity regulating kinase family members, causing microtubule disruption and tau toxicity [15]. In relation to this, Pei et al. further discovered that DAPK1 could also directly phosphorylate tau at the Ser262 residue by interacting with the microtubule repeat domain of tau proteins [16]. These findings clearly substantiate a pivotal role of the DAPK1/Pin1 pathway in modulating tau function in neurons. Although $A \beta$ oligomers are able to stimulate tau phosphorylation, it is unclear whether DAPK1 is involved in this process.
The protein stability of DAPK1 can be regulated by the ubiquitin-proteasome system by binding to heat shock protein 90 (HSP90) or DAPK-interacting protein $1[17,18]$. HSP90 has a fundamental role in regulating cellular stress responses through maintaining protein homeostasis. HSP90 forms complexes with DAPK1 by recognizing specific amino acid residues in the kinase domain [18]. The complexation not only contributes to DAPK1 stabilization, but also facilitates its activation [18]. Importantly, studies have demonstrated that HSP90 protein levels are upregulated in brains of $\mathrm{AD}$ patients [19]. HSP90 inhibitors not only attenuate tau and $A \beta$ pathologies, but also promote the proteasomal degradation of DAPK1 [18, 20]. However, whether $A \beta$ oligomers could regulate DAPK1 function through HSP90 remains to be studied.

In the present study, we aimed to clarify whether $A \beta$ oligomers could modulate tau phosphorylation by influencing DAPK1 function. In detail, we reported that soluble $A \beta$ aggregates stabilized the DAPK1 protein through HSP90, leading to subsequent Pin1 dysfunction by phosphorylating the Ser71 residue. This further caused tau accumulation and hyperphosphorylation at multiple AD-related sites, as well as caspase-3 dependent apoptosis in primary neurons. Genetic or chemical deactivation of DAPK1 protected neurons against $A \beta$ species-induced neuronal apoptosis and tau dysregulation. Our study implies a mechanistic link between $\mathrm{A} \beta$ and tau pathologies via DAPK1.

\section{Materials and Methods}

\section{Chemicals and reagents}

Synthetic human $A \beta$ (1-42) peptides (A $\beta 42)$ were purchased from Chinapeptide (Shanghai, China). The DAPK1-specific inhibitor (4Z)-4-(3-pyridylmethylene)-2-styryl-oxazol-5-one (C6) was acquired from Calbiochem (California, United States). The HSP90 inhibitor 17-allylamino-17-demethoxygeldanamycin (tanespimycin, 17-AAG) was from Macklin (Shanghai, China). DMSO, trypan blue powder, 1, 1, 1, 3, 3, 3-hexafluoro-isopropanol (HFIP), cycloheximide (CHX), poly-D-lysine and cytosine $\beta$-D-arabinofuranoside were purchased from Sigma-Aldrich (Missouri, United States). 3-(4, 5-Dimethylthiazol2-yl)-2,5-diphenyltetrazolium bromide (MTT), nonfat milk powder and bovine serum albumin (BSA) were from Sangon (Shanghai, China). Hoechst 33342 solution was purchased from Beyotime (Shanghai, China). Fetal bovine serum (FBS), Dulbecco's modified Eagle's medium (DMEM), sodium pyruvate, GlutaMAX ${ }^{\mathrm{TM}}$ Supplement, Hank's balanced salt 
solution (HBSS), glucose solution, trypsin and penicillin-streptomycin were provided by Gibco (Texas, United States). Neurobasal medium and the B-27 supplement were obtained from BasalMedia (Shanghai, China). The anti-phospho-Ser71-Pin1 (pS71-Pin1) antibody was prepared and purified by immunizing rabbits with a peptide sequence (CSQSRRPSSWR) of Pin1 containing the pS71 residue (Abmart, Shanghai, China).

\section{Animals}

Pregnant wild type (WT) C57BL/6 mice were purchased from Shanghai Laboratory Animal Research Center and were kept with food and water supply until the delivery of pups. The production of DAPK1 knockout (KO) mice (on a C57BL/6 background) was described previously [21]. All mice were maintained in the SPF facility of Fujian Medical University with a 12/12 h light/dark cycle and ad libitum access to food. All animal experiments were approved by the Animal Ethics Committee of Fujian Medical University.

\section{Primary neuron culture}

Primary neurons from $\mathrm{WT}$ and DAPK1 $\mathrm{KO}$ mice were prepared according to previous studies $[22,23]$, with minor modifications. Briefly, P0 pups were decapitated for cortical tissues. Cells were isolated and resuspended in DMEM supplemented with $10 \%$ FBS, $100 \mathrm{U} / \mathrm{ml}$ penicillin, $100 \mu \mathrm{g} / \mathrm{ml}$ streptomycin and sodium pyruvate. Cells were then plated onto poly-D-lysine-coated plates. After $4 \mathrm{~h}$ of culture at 37 ${ }^{\circ} \mathrm{C}$ and $5 \% \mathrm{CO}_{2}$, the medium was replaced with neurobasal medium containing 2\% B-27, GlutaMAX supplement, $100 \mathrm{U} / \mathrm{ml}$ penicillin and $100 \mu \mathrm{g} / \mathrm{ml}$ streptomycin. Cytosine- $\beta$-D-arabinofuranoside was added to the medium at 3 days in vitro (DIV3) to obtain neuronal-enriched cultures. Cells were maintained at $37{ }^{\circ} \mathrm{C}$ and $5 \% \mathrm{CO}_{2}$ by replacing half of the medium with fresh neurobasal medium every two days. Cultures were used at DIV7-9 for experiments.

\section{Preparation and characterization of $A \beta 42$ species}

The preparation of $\mathrm{A} \beta 42$ species in different aggregation states was performed according to Stine et al. [24, 25]. In brief, synthetic A $\beta 42$ peptides (1 mg) were first dissolved in $250 \mu \mathrm{L}$ of cold HFIP and incubated at room temperature overnight. HFIP was then removed using a lyophilizer (SP Scientific, Pennsylvania, United States). Afterward, A $\beta 42$ peptides were reconstituted using DMSO to obtain a stock concentration of $5 \mathrm{mM}$. To prepare LMW A $\beta$ species, the A $\beta 42$ stock was diluted with cold PBS to a final concentration of $100 \mu \mathrm{M}$. The PBS solution was incubated at $4{ }^{\circ} \mathrm{C}$ for $24 \mathrm{~h}$. To obtain HMW A $\beta$ species, the stock was diluted using $10 \mathrm{mM} \mathrm{HCl}$ solution to $100 \mu \mathrm{M}$ and was subsequently incubated at $37^{\circ} \mathrm{C}$ for $24 \mathrm{~h}$. The aggregation states of both samples were examined using tris-tricine gel $(15 \%)$ electrophoresis and the 6E10 antibody (BioLegend, California, United States). To validate the presence of $A \beta$ oligomers, we also conducted dot blot analysis using an anti-oligomer A11 antibody (Thermo Fisher, Massachusetts, United States). The preparation of A $\beta 42$ species was frequently checked to maintain the quality of $A \beta$ used in the experiments.

\section{MTT assay}

To examine the cytotoxicity of $\mathrm{A} \beta 42$ species, primary neurons were seeded in poly-D-lysine-coated 96-well plates $\left(5 \times 10^{4}\right.$ cells/well). At DIV7, cells were treated with different concentrations of HMW or LMW A $\beta 42$ species $(0.5,2,10$ and $20 \mu \mathrm{M}$, equivalent to monomers) or vehicle for $24 \mathrm{~h}$. MTT stock (5 $\mathrm{mg} / \mathrm{mL}$ in PBS) was added into each well to a working concentration of $0.5 \mathrm{mg} / \mathrm{mL}$. After $4 \mathrm{~h}$ of incubation at $37^{\circ} \mathrm{C}$ and $5 \% \mathrm{CO}_{2}$, the cell medium was removed, and DMSO at $100 \mu \mathrm{L} /$ well was added to dissolve formazan crystals. The plate was shaken for 10 min and subjected to absorbance measurements at $570 \mathrm{~nm}$ and $630 \mathrm{~nm}$ using a microplate reader (Thermo Fisher). All samples were prepared in triplicate.

\section{Trypan blue staining}

To quantify neuronal cell death caused by $\mathrm{A} \beta 42$ species, primary neurons were seeded in coated 48 -well plates $\left(5 \times 10^{4}\right.$ cells/well). At DIV7, cells were treated with different concentrations of LMW or HMW A $\beta 42$ species or vehicle for $24 \mathrm{~h}$. The trypan blue stock solution ( $0.4 \%$ in PBS) was diluted 10 -fold using PBS. Trypan blue solution was added to the cells (300 $\mu \mathrm{L} /$ well), and the plate was incubated at room temperature for $5 \mathrm{~min}$. The solution was replaced with PBS and cells were imaged under a microscope (Zeiss, Oberkochen, Germany). At least three different fields were recorded for the same sample. The images were analyzed using ImageJ software (version 1.50i, NIH, United States) to count live and dead cells.

\section{TUNEL assay}

To further determine $A \beta 42$-induced cell death, we carried out a TUNEL assay using an in situ cell death detection kit, TMR red (Roche, Indiana, United States), following the manufacturer's manual. Primary neurons $\left(5 \times 10^{4}\right.$ cells/well) were seeded in 24-well plates containing poly-D-lysine-coated coverslips (EMS, Pennsylvania, United States). At DIV7, neurons were treated with various concentrations of LMW or HMW A $\beta 42$ species or 
vehicle for $24 \mathrm{~h}$. TUNEL reagents were added to each well after cell fixation and permeabilization, and were incubated with cells at $37^{\circ} \mathrm{C}$ for $1 \mathrm{~h}$. After washing with PBS, cells were incubated with Hoechst 33342 (1:2000 dilution) to stain nuclei. All samples were imaged using a fluorescence microscope (Zeiss). At least three different fields were recorded for each sample. The data were evaluated with ImageJ (version $1.50 \mathrm{i})$ to quantify TUNEL-positive cells.

\section{Immunoblot analysis}

Primary neurons were seeded in poly-D-lysine-coated 6-well plates at a density of $8 \times 10^{5}$ cells/well. At DIV7, cells were treated with either $A \beta 42$ species alone or together with inhibitors, as indicated in each experiment. After $24 \mathrm{~h}$, the cells were harvested and lysed using radioimmunoprecipitation assay buffer (RIPA buffer) in the presence of protease and phosphatase inhibitor cocktails (Transgene, Beijing, China). The protein concentration was determined using a BCA protein assay kit (Beyotime). Protein samples (10-15 $\mu \mathrm{g})$ were separated by SDS-PAGE and transferred to $0.45-\mu \mathrm{m}$ polyvinylidene fluoride membranes (Millipore, Massachusetts, United States). Following transfer, the membranes were blocked with 5\% BSA-TBST or 5\% milk-TBST at room temperature for $1 \mathrm{~h}$. The membranes were probed with primary antibodies and HRP-conjugated secondary antibodies. Target proteins were further detected using ECL chemiluminescent HRP substrate (Millipore) in a Bio-Rad Chemidoc imaging system (California, United States). All blots were analyzed using the Image software (version 1.50i) to determine the optical densities. Data were normalized to the corresponding $\beta$-actin bands. The primary antibodies used in the study can be found in the Supplementary Information (Table S1).

\section{RNA extraction and $q P C R$}

Whole RNA was isolated from primary neurons treated with $A \beta$ species or vehicles using NucleoZOL (Macherey-Nagel, Dueren, Germany). The cDNA was first synthesized using HiScript II Q RT SuperMix (Vazyme, Nanjing, China). qPCR assays were then performed using ChamQ Universal SYBR qPCR Master Mix (Vazyme) in a QuantStudio Real-Time PCR system (Applied Biosystems, Massachusetts, United States). Data were analyzed with the comparative Ct $(\Delta \Delta \mathrm{Ct})$ method by normalizing the value to the 18S ribosomal RNA (rRNA) or GAPDH level. The primer sequences used in the study were as follows: mouse DAPK1, forward 5'-GCACCCAAA TGTCATCACCCT-3', reverse 5'-AAACAGCTCACC TCCTGCAAC-3'; 185 rRNA, forward 5'-TGTCTC
AAAG-ATTAAGCCATGCA-3', reverse 5'-GCGACC AAAGGA-ACCATAACTG-3'; GAPDH, forward 5'-AGGTCGGTGTGAACGGATTTG-3', reverse 5'TGTAGACCATG-TAGTTGAGGTCA-3'.

\section{DAPK 1 protein stability assay}

The protein stability of DAPK1 in primary neurons was measured by incubating neurons (DIV7) with CHX $(10 \mu \mathrm{g} / \mathrm{ml})$ in the absence or presence of A 342 species for indicated times. Samples were collected at 0, 3, 6 and $12 \mathrm{~h}$ after $\mathrm{CHX}$ addition, and were subjected to immunoblot analysis to probe the protein level of DAPK1.

\section{Statistical analysis}

Statistical analyses were conducted using the GraphPad Prism software (version 8.3.0, GraphPad, California, United States). Data were collected from at least three independent cell cultures as indicated in the figure legends. No test for outliers was conducted. The Shapiro-Wilk test was used for normality test. All data are expressed as mean \pm standard deviation (SD). The statistical significance was analyzed by either two-tailed unpaired $t$-test or one-way ANOVA followed by Tukey's post hoc test. $p$ value $<0.05$ was considered significant.

\section{Results}

\section{Characterization of $A \beta 42$ species}

The preparation of $A \beta$ aggregates from synthetic $A \beta 42$ peptides has been well described in the literature. By changing the incubation conditions (e.g., ionic strength and $\mathrm{pH}), \mathrm{A} \beta$ species in different aggregation states can be formed. We followed the protocols developed by Stine et al. [25] and sought to prepare $A \beta 42$ oligomers and fibrils (Figure 1A). The distribution of $A \beta 42$ species was first examined using tris-tricine gel electrophoresis and the 6E10 antibody (Figure 1B). Under physiological $\mathrm{pH}$ and ionic strength and low temperature $\left(4^{\circ} \mathrm{C}\right), \mathrm{A} \beta 42$ mainly formed LMW species between 10 and $15 \mathrm{kDa}$, corresponding to trimeric and tetrameric $A \beta 42$ species. The dominant species below $10 \mathrm{kDa}$ were monomers either from unaggregated $A \beta$ materials or the dissociation of $A \beta$ aggregates, or both. An additional band can be observed between the monomer and trimer bands, likely representing $A \beta 42$ dimers $(\sim 9 \mathrm{kDa})$. When incubated at acidic $\mathrm{pH}$ and low ionic strength $(10 \mathrm{mM} \mathrm{HCl})$ at $37{ }^{\circ} \mathrm{C}, \mathrm{A} \beta 42$ peptides also formed dimers, trimers and tetramers, as depicted by multiple bands below $15 \mathrm{kDa}$. Moreover, we observed a smear between 60 and 180 $\mathrm{kDa}$, and a strong signal at the top of the membrane, suggesting the formation of large $\mathrm{A} \beta 42$ aggregates and fibrils with high molecular weight after 
incubation. The large assemblies were not visible in A 342 samples incubated at $4{ }^{\circ} \mathrm{C}$ and were termed HMW species. The size distributions of $A \beta 42$ assemblies in our study were in accordance with those reported by Dahlgren et al. [26]. To further validate the presence of pre-fibrillar oligomers in samples, we performed dot blot analysis using an oligomer-specific A11 antibody that recognizes $A \beta$ species ranging from trimeric to $\sim 75 \mathrm{kDa}$ [27]. As seen from the data (Figure 1C), A $\beta 42$ aggregates from both preparations contained A11-positive species in solution. Since A11-positive oligomers are also present in brains of AD patients, we carried out MTT assays to evaluate the cytotoxicity of $A \beta 42$ species prepared in our study (Figure 1D). Primary neurons were incubated with various concentrations (equivalent to monomers) of $\mathrm{A} \beta 42$ for $24 \mathrm{~h}$. LMW A $\beta 42$ species induced a significant decrease in cell viability at $10 \mu \mathrm{M}$, while HMW A $\beta 42$ species markedly reduced cell viability at $0.5 \mu \mathrm{M}$ and manifested a concentration-dependent toxic effect on primary neurons. Thus, we successfully prepared two types of $A \beta 42$ species with partially overlapping compositions. These species contained physiologically relevant $A \beta$ aggregates and were neurotoxic in vitro.

\section{A 342 species activate DAPK 1 function in primary neurons}

We next studied whether the prepared $A \beta 42$ species were able to regulate the function of DAPK1 in primary neurons. Our previous research has demonstrated that DAPK1 could directly interact with Pin1 and phosphorylate its Ser71 residue [14]. Therefore, we determined the level of pS71-Pin1 to monitor the enzymatic activity of DAPK1 upon A $\beta 42$ treatment. Both LMW and HMW A $\beta 42$ species were able to upregulate DAPK1 protein levels, especially at a concentration of $20 \mu \mathrm{M}$ (Figure 2A, B, D and G), without affecting its mRNA level (Figure $2 \mathrm{C}$ and Figure S1). Corresponding to the elevation in DAPK1 expression, Pin1 phosphorylation at the Ser71 residue was also significantly increased in primary neurons incubated with $20 \mu \mathrm{M}$ A $\beta 42$ species (Figure 2A, B, E and $\mathrm{H}$ ), whereas the total Pin1 level remained constant among neurons treated with or without

\section{A}

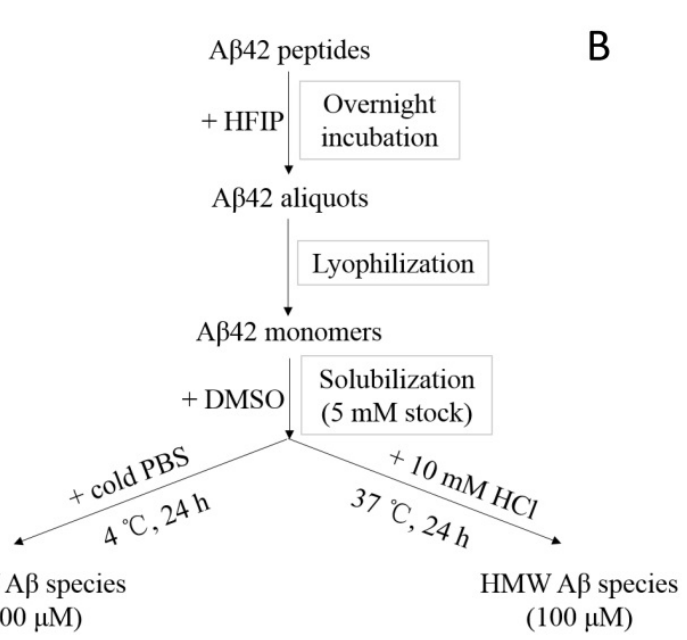

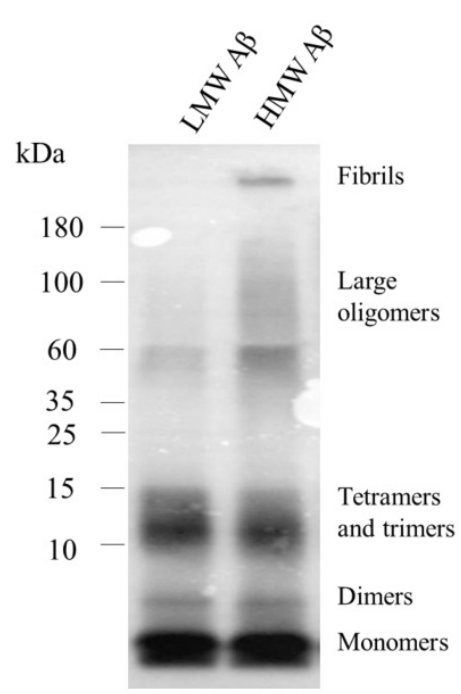

C

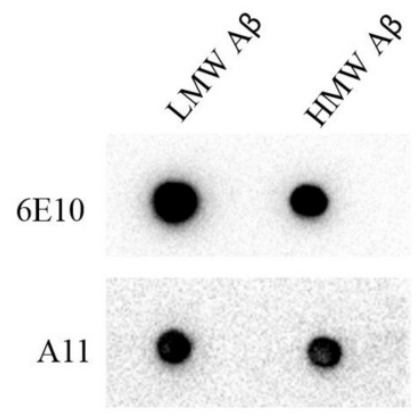

D

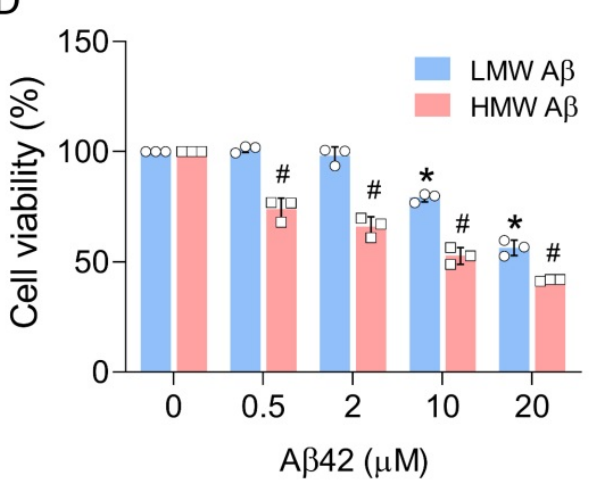

Figure 1. Preparation and characterization of $A \beta 42$ species. (A) The protocol used for the preparation of LMW and HMW AB42 species from synthetic peptides. (B) Tris-tricine gel analysis of the size distribution of LMW and HMW AB42 species as probed by the 6E10 antibody. (C) Dot blot analysis of the presence of $A 11$-positive species in both preparations. (D) MTT assay to measure the cytotoxicity of LMW and HMW A 342 species in mouse primary neurons. Data are expressed as the mean \pm SD (* and \#, $p<0.05$ vs. $0 \mu \mathrm{M})$. 
$\mathrm{A} \beta 42$ species (Figure $2 \mathrm{~A}, \mathrm{~B}, \mathrm{~F}$ and I). The increase in pS71-Pin1 levels clearly supported that $A \beta 42$ species could enhance the function of DAPK1. Furthermore, our results indicated that $A \beta$ aggregates upregulate DAPK1 expression through a transcriptionindependent mechanism. In addition, both $A \beta$ preparations showed similar effects based on the quantification, suggesting that DAPK1 activation might be a common pathway in mediating $A \beta$ aggregation-related cytotoxicity.

\section{DAPK 1 activation mediates $A \beta 42$ species-induced neuronal apoptosis}

Since DAPK1 plays an important role in regulating cell survival under stress response, we further determined whether DAPK1 activation by A $\beta 42$ species could result in neuronal cell death. We first applied a TUNEL assay to detect cell death in primary neurons. It is evident from the fluorescence imaging that TUNEL positive cells were increased dramatically with the addition of $\mathrm{A} \beta 42$ species (Figure 3A). Approximately $50 \%$ of cells died in WT neurons treated with $20 \mu \mathrm{M}$ A $\beta 42$ species, irrespective of the aggregation state (Figure $3 \mathrm{~B}$ and $\mathrm{C}$ ). In line with the TUNEL assay, trypan blue staining also showed that around $50 \%$ of WT neurons incorporated the dye in the presence of $20 \mu \mathrm{M} \mathrm{A} \beta 42$ species (Figure S2), pointing to substantial cell death caused by the treatment. However, none of the $A \beta 42$ species were capable of triggering significant cell death in DAPK1 $\mathrm{KO}$ neurons, as shown in the TUNEL assay (Figure $3 \mathrm{~A}, \mathrm{~B}$ and $\mathrm{C})$, indicating a protective effect of DAPK1 ablation on $A \beta$-induced neuronal loss. To better understand how $A \beta 42$ species lead to neuronal cell death, we evaluated apoptosis markers that are closely associated with DAPK1 activation. Consistent with the DAPK1 increase after $A \beta 42$ species treatment (Figure 3D, E, H and I), levels of cleaved caspase-3 and cleaved poly-ADP-ribose polymerase-1 (PARP1) were markedly increased in neurons treated with 20 $\mu \mathrm{M} A \beta 42$ aggregates (Figure 3D and $\mathrm{H}$ ), suggesting that $A \beta 42$ induces neuronal cell death through caspase-3 dependent apoptosis. Moreover, we examined whether ablating DAPK1 protein was able to protect neurons against apoptosis caused by $A \beta 42$ species. Although DAPK1 KO fully reversed HMW
A

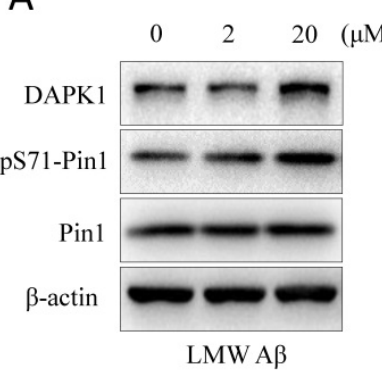

D LMW A $\beta$

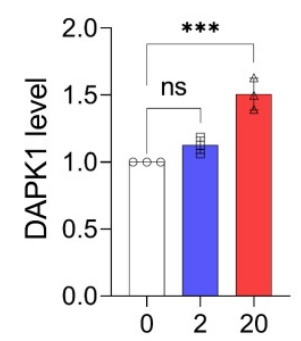

G

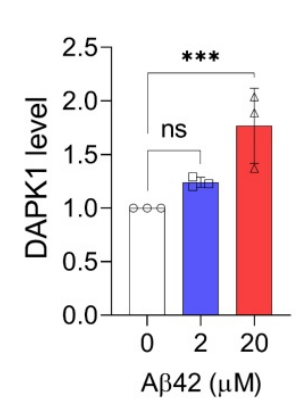

B

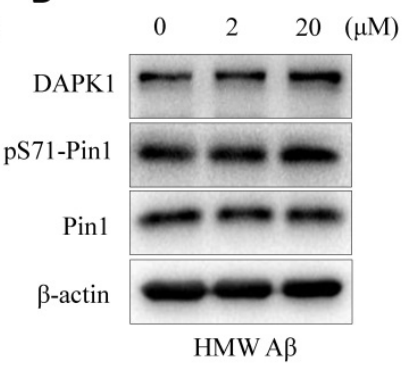

$\mathrm{E}$

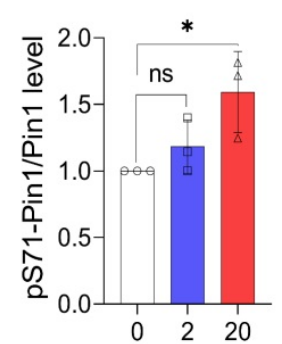

$\mathrm{H}$

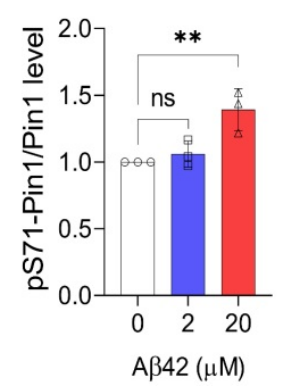

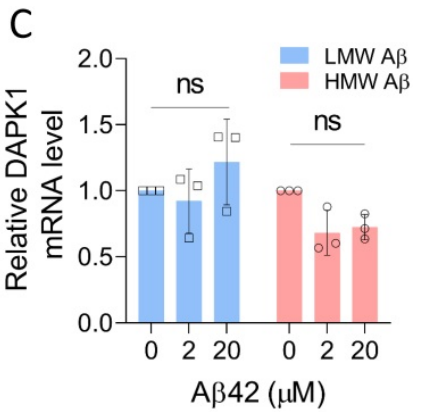

$\mathrm{F}$

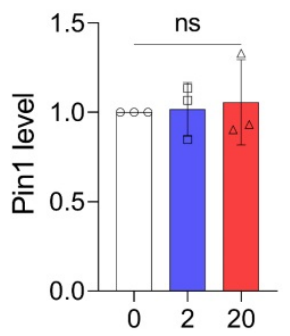

I

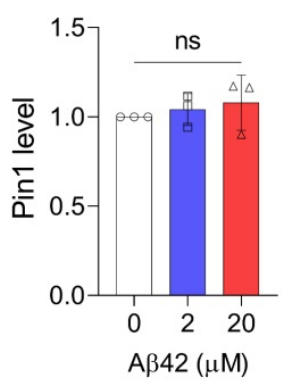

Figure 2. A 442 species upregulate DAPK1 protein levels and function. (A) and (B) Immunoblot analysis of DAPK1, pS71-Pin1 and Pin1 levels in primary neurons treated with different concentrations of LMW and HMW A 42 for $24 \mathrm{~h}$. (C) Real-time PCR measurement of DAPK1 mRNA levels in WT primary neurons treated with different concentrations of LMW and HMW A 42 species. 18S ribosomal RNA was used as an internal control. The corresponding statistics for immunoblot analysis are shown in (D), $(\mathrm{E})$ and $(\mathrm{F})$ and $(\mathrm{G}),(\mathrm{H})$ and $(\mathrm{I})$. $\beta$-actin was used as an internal control. Data are expressed as the mean \pm SD $\left({ }^{*} p<0.05\right.$, ${ }^{* *} p<0.01, * * * p<0.001$, ns, not significant). 
$\mathrm{A} \beta$ species-induced caspase- 3 activation and PARP cleavage (Figure $3 \mathrm{H}, \mathrm{J}$ and $\mathrm{K}$ ), it only partially prevented LMW $A \beta$-induced apoptotic changes in primary neurons (Figure 3D, F and G). However, reduced cell death along with the decrease in cleaved caspase-3 and PARP1 levels in DAPK1 KO neurons treated with $A \beta 42$ species substantiates the critical role of DAPK1 in mediating A $\beta 42$ species-induced neuronal apoptosis.

\section{A 342 species promote tau accumulation and hyperphosphorylation by activating DAPK 1}

The interplay between $A \beta$ aggregation and tau dysregulation is essential for understanding the pathoetiology of $\mathrm{AD}$, as well as for developing disease-modifying treatments. Previous studies have indicated that soluble $A \beta$ species could not only promote tau phosphorylation by regulating protein kinases such as CDK5 and GSK-3 $\beta$ [28], but also enhance the intracellular aggregation and intercellular spreading of tau proteins $[29,30]$. Pin1 could regulate the protein stability of tau and its phosphorylation state, which are both crucial for maintaining the physiological function of tau in neurons [31]. It has been shown that DAPK1 activation triggers aberrant tau phosphorylation in AD and stroke $[12,16]$, while whether DAPK1 and Pin1 also participate in A $\beta$ species-induced tau dysregulation remains to be determined. We therefore first compared the phosphorylation of Pin1 at the Ser71 residue (pS71-Pin1) in WT and DAPK1 KO primary neurons after $A \beta 42$ species treatment. As shown in figure 4 , all neurons had comparable Pin1 levels. WT neurons treated with $\mathrm{A} \beta 42$ species exhibited a significant increase in pS71-Pin1 levels, while this increase was diminished in DAPK1 KO neurons exposed to A $\beta 42$

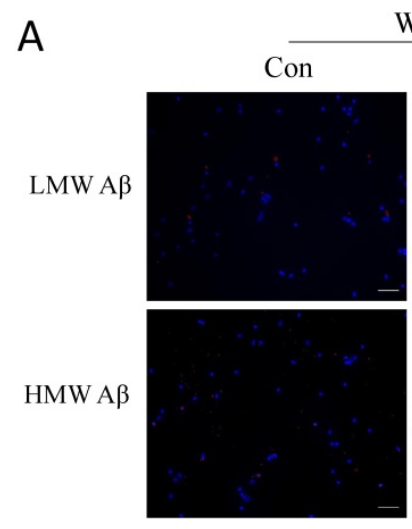

WT
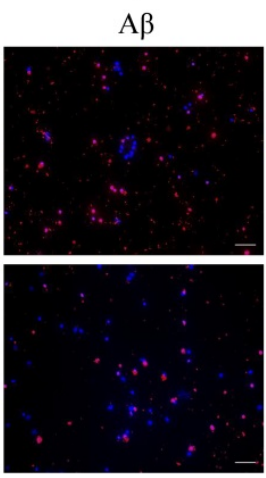

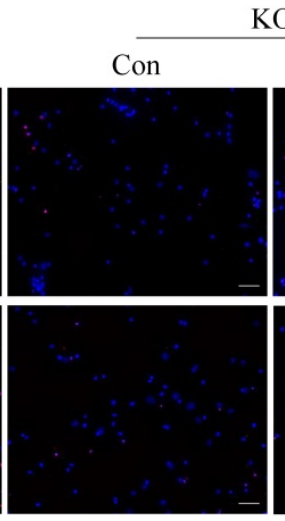

$\mathrm{KO}$

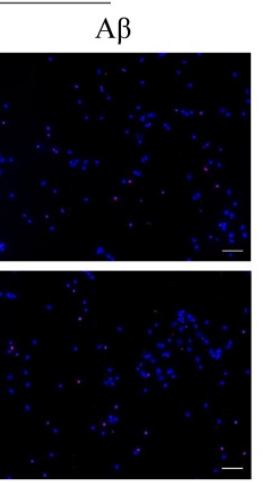

$\mathrm{B}$

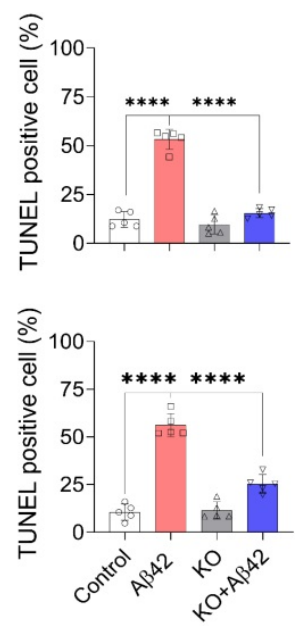

D

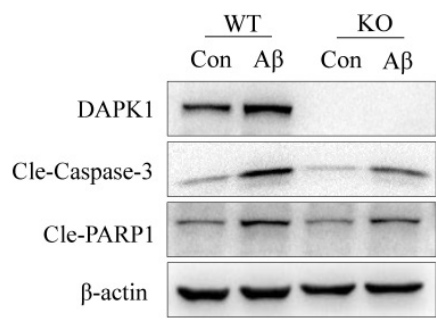

$\mathrm{H}$

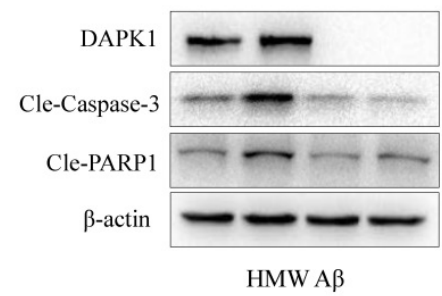

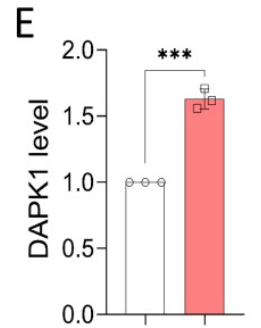

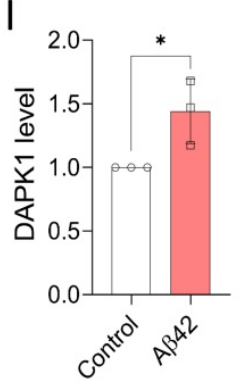

$\mathrm{F}$

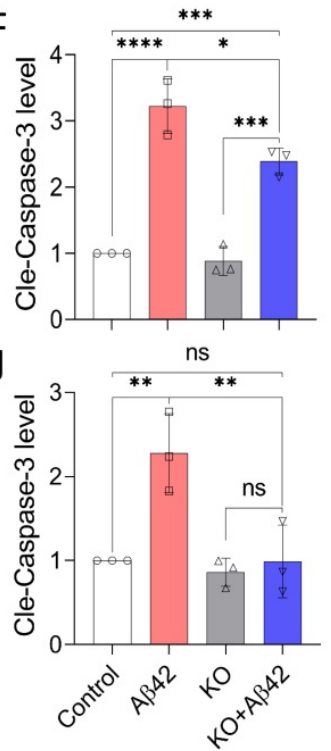

G
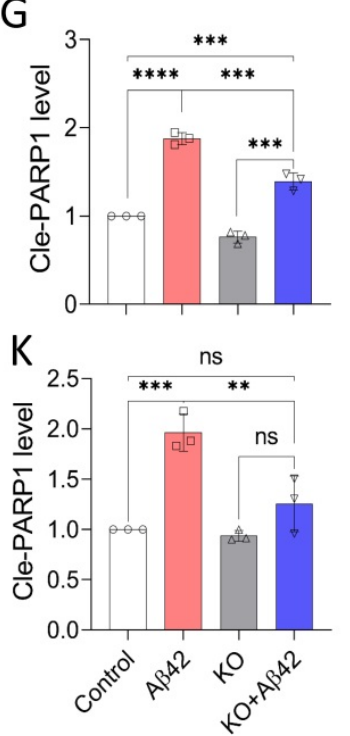

Figure 3. DAPK1 activation mediates AB42 species-induced neuronal apoptosis. (A) TUNEL assay showing cell death of WT or DAPK1 KO primary neurons treated with or without $20 \mu \mathrm{M} \mathrm{A \beta 42} \mathrm{species} \mathrm{for} 24 \mathrm{~h}$. Blue staining indicates cell nuclei and magenta staining represents TUNEL-positive cells. Scale bar $50 \mu \mathrm{m}$. (B) and (C) Statistical analysis of

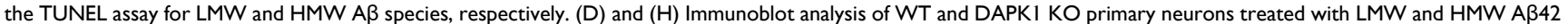
species $(20 \mu \mathrm{M})$ for $24 \mathrm{~h}$ to detect DAPK1, cleaved caspase-3 and cleaved-PARPI levels. (E), (F) and (G) and (I), (J) and (K) are the corresponding statistics. $\beta$-actin was used as an internal control. Data are expressed as the mean \pm SD $(* p<0.05, * * p<0.01, * * * p<0.001, * * * * p<0.0001$, ns, not significant). 
(Figure 4A and B). Pin1 phosphorylation at Ser71 has been known to negatively regulate its substrate binding affinity and isomerization activity, thereby decelerating the conformational conversion of cis-ptau to trans-ptau, which is essential for maintaining tau homeostasis $[32,33]$. We further detected total and phospho-tau levels in WT and DAPK1 KO samples. Corresponding to the change in pS71-Pin1 levels, both the total tau and tau phosphorylation at AD-related sites, including Thr231, Ser262 and Ser396, were remarkably elevated in WT neurons incubated with $\mathrm{A} \beta 42$ species, whereas they were not affected by $A \beta 42$ treatment in DAPK1 KO primary neurons (Figure 4A and $B$ ). This result confirmed our previous report that DAPK1 activation leads to abnormal tau accumulation by inactivating Pin1 function [12]. Our findings also hinted that LMW and HMW A $\beta 42$ species showed similar effects on tau accumulation and phosphorylation. These results reveal for the first time that DAPK1 activation indeed participates in A $\beta 42$ species-induced tau dysregulation and support a mechanistic link between $A \beta$ and tau pathologies in the progression of $\mathrm{AD}$.

\section{The DAPK 1-specific inhibitor $\mathrm{C} 6$ protects neurons against $A \beta 42$ species-induced apoptosis and tau dysregulation}

Because of the beneficial effect of DAPK1 KO against $A \beta 42$ species-induced cell death and tau phosphorylation, we further investigated the impact of a DAPK1-specific inhibitor on A 442 -induced neuronal damage to confirm the efficacy of DAPK1 inhibition in AD. The compound C6 (4-(pyridin-
3-ylmethylene)oxazol-5(4H)-one) is a selective and potent inhibitor of DAPK1 and has been used as a potential treatment for ischemic brain injury in mouse models [34, 35]. We first verified that $2 \mu \mathrm{M}$ C6 treatment was able to suppress DAPK1 activity, as indicated by a significant increase in pSer308-DAPK1 levels (Figure S3). As shown in figure 5A and B, C6 treatment significantly attenuated $\mathrm{A} \beta 42$ speciesinduced Pin1 phosphorylation at Ser71 without affecting Pin1 levels, further confirming the inhibition of DAPK1 function by the compound. In parallel with DAPK1 inhibition, the activation of caspase- 3 and the cleavage of PARP1 were significantly ameliorated by treatment with $\mathrm{C} 6$ in neurons exposed to $\mathrm{A} \beta 42$ species (Figure 5A and $\mathrm{B}$ ), implicating an improvement in A $\beta 42$-induced neuronal apoptosis following C6 treatment. The impact of $\mathrm{C} 6$ on $\mathrm{A} \beta 42$ species-induced tau accumulation and hyperphosphorylation was also assessed using an immunoblotting assay. The level of total tau protein in cells treated with both $A \beta 42$ species and C6 was similar to that of the control samples (Figure 5C and D). In agreement with the reduction in total tau level, C6 treatment entirely reversed HMW A $\beta 42$ species-induced tau phosphorylation at the Thr231, Ser262 and Ser396 residues (Figure 5D). DAPK1 inhibition by C6 was also capable of fully blocking the elevation of pS262 and pS396-tau caused by LMW A $\beta 42$; however, the increase in the pT231-tau level was only partially reduced by the compound compared with neurons treated with $\mathrm{A} \beta 42$ alone $(p<0.05)$ (Figure 5C). These results again indicate that inhibiting DAPK1 activity confers protective effects on $A \beta$ aggregation-induced
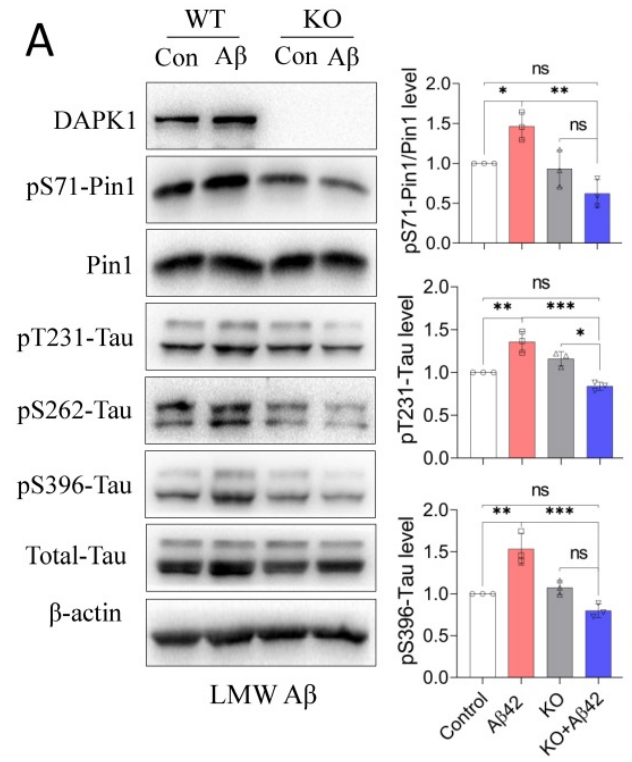

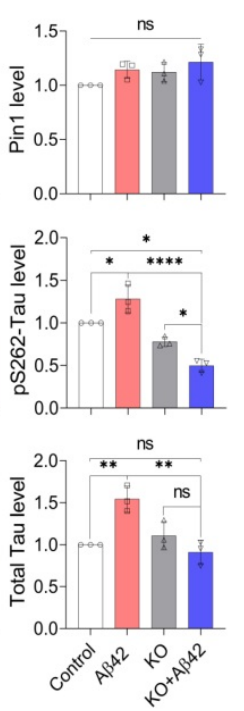

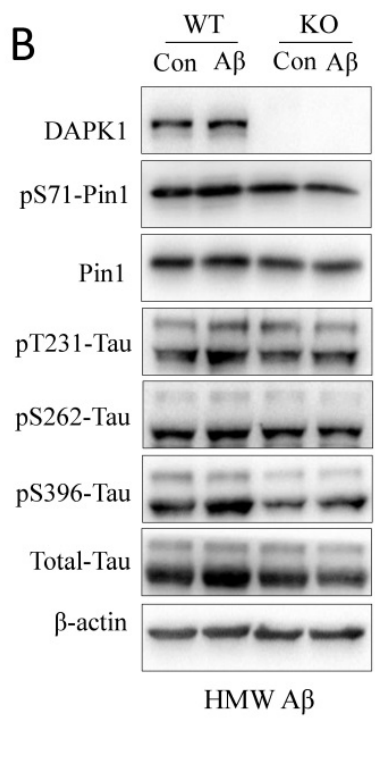
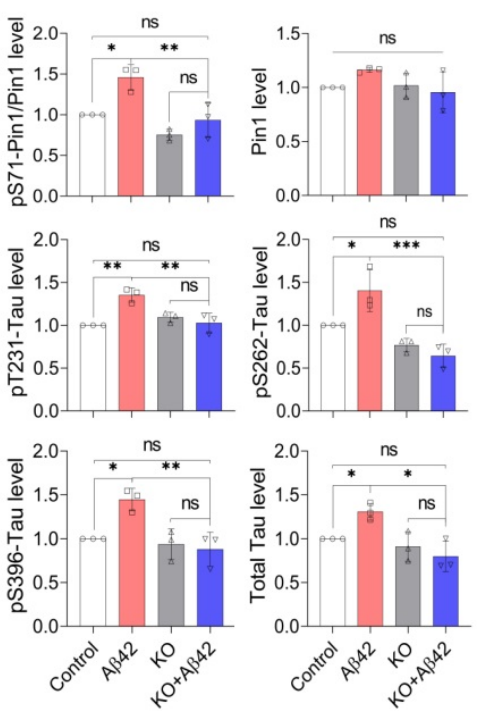

Figure 4. A 342 species induce tau accumulation and hyperphosphorylation via the DAPK1/Pin l pathway. (A) and (B) Immunoblot analysis and statistics of WT and DAPK 1 KO primary neurons treated with LMW or HMW A 42 species $(20 \mu \mathrm{M})$ for $24 \mathrm{~h}$ to determine the levels of DAPK1, pS71-Pin 1, Pin 1, total tau and tau phosphorylation at the Thr231, Ser262 and Ser396 residues. $\beta$-actin was used as an internal control. Data are expressed as the mean \pm SD $(* p<0.05$, $* * p<0.01$, $* * * p<0.001$, $* * * * p<0.0001$, ns, not significant). 
neuronal damage. Together with findings from DAPK1 KO primary neurons, inhibition of DAPK1 function provides significant protection against $A \beta 42$ aggregation-induced cell apoptosis and tau dysregulation, further corroborating the pivotal role of DAPK1 in modulating the key pathologies of AD.

\section{A 342 species increase the protein stability of DAPK 1 by modulating HSP90 function}

Because DAPK1 mRNA levels were not significantly altered by $A \beta 42$ species (Figure $2 C$ ), to examine the regulatory effect of $A \beta$ on DAPK1 expression, we then assessed the protein stability of DAPK1 in neurons following A 342 species treatment using the $\mathrm{CHX}$ assay. According to figure $6 \mathrm{~A}$ and $\mathrm{B}$, DAPK1 protein was degraded over time in the presence of $\mathrm{CHX}$ treatment, while primary neurons incubated with $\mathrm{A} \beta 42$ had a slower DAPK1 degradation rate compared with neurons treated with vehicle. At $12 \mathrm{~h}$, the level of DAPK1 in the A $\beta 42$ group was also significantly higher than that in the vehicle group. Therefore, we concluded that the upregulation of DAPK1 after A $\beta 42$ treatment is likely a result of reduced DAPK1 degradation. Due to the close association between HSP90 and DAPK1 stability and the elevation of HSP90 levels in the brains of AD patients $[10,19]$, we hypothesized that $A \beta 42$ induced DAPK1 upregulation might be mediated by HSP90. To prove this, we coincubated primary neurons with A 342 species and tanespimycin (17-AAG), a potent HSP90 inhibitor, and detected whether A $\beta 42$ was still able to increase DAPK1 protein levels. As evident from figure $6 \mathrm{C}$ and $\mathrm{D}, 17-\mathrm{AAG}$ alone had a limited effect on DAPK1 expression, while it could significantly block A $\beta 42$ species-induced DAPK1 elevation, implicating a causative role of HSP90 in modulating A $\beta 42$-stimulated DAPK1 upregulation. Interestingly, we did not observe any change in HSP90 protein levels among all samples (Figure 6C and $\mathrm{E})$; however, it has been suggested that the cellular function of HSP90 might be activated by $\mathrm{A} \beta$ aggregates as a result of the unfolded protein response [36]. Along with DAPK1 reduction, 17-AAG also robustly decreased $A \beta 42$-induced tau accumulation (Figure 6C and F), further verifying the function of DAPK1 in connecting $A \beta$ aggregation and tau pathologies. Thus, our results indicate that the activation of HSP90 function by A $\beta$ aggregates contributes to DAPK1 stabilization, whereby $\mathrm{A} \beta$ species further lead to neuronal apoptosis and aberrant tau accumulation (Figure 7).

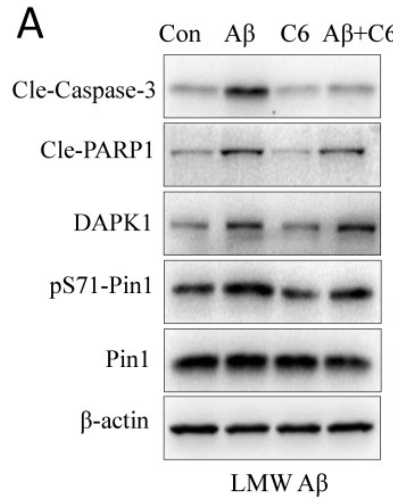

C

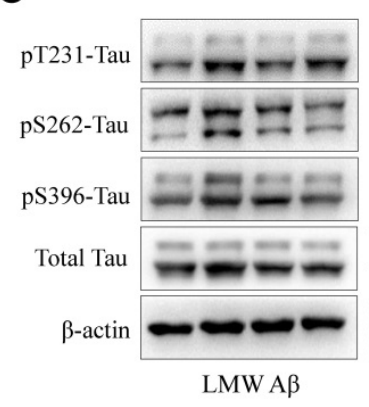

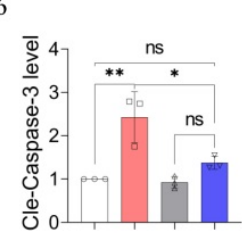
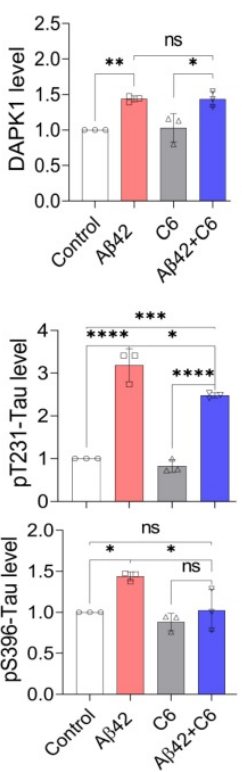
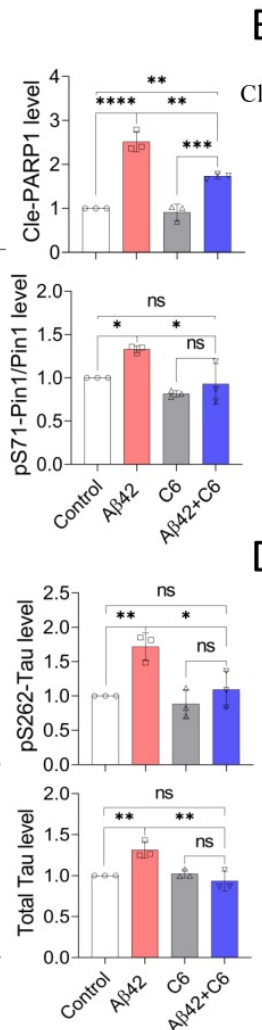

B

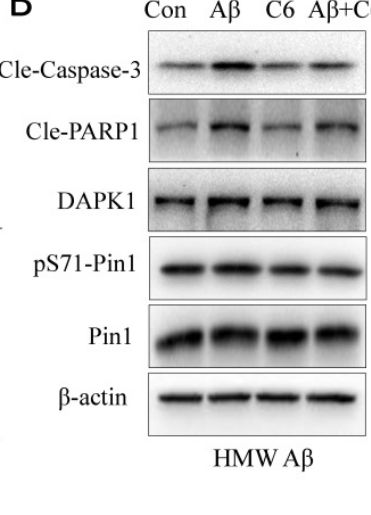

D

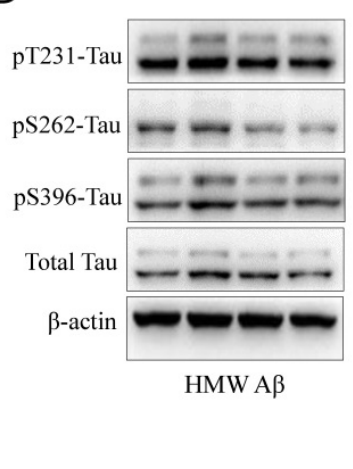

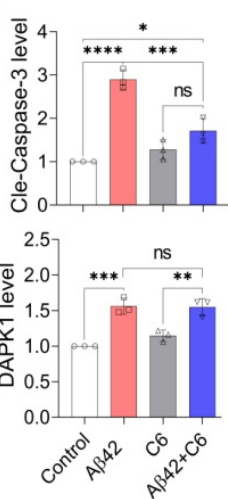
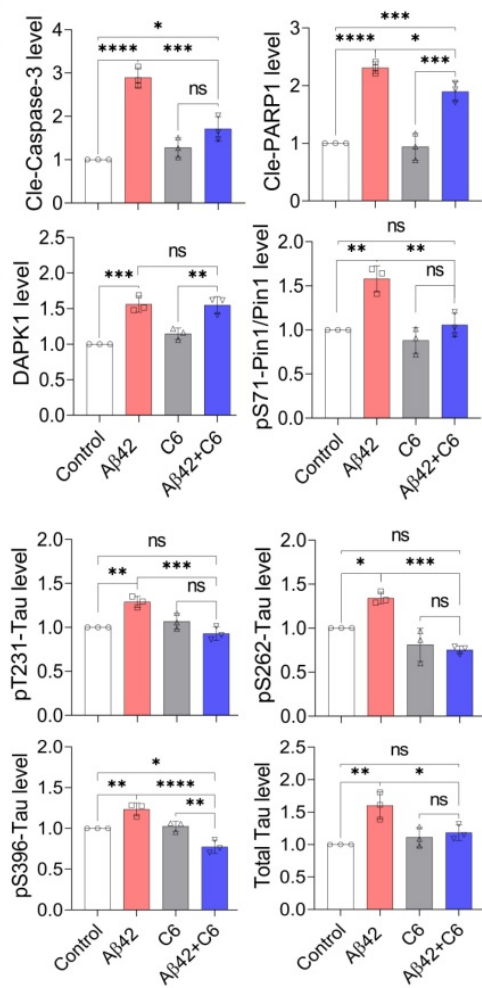

Figure 5. DAPK1 inhibition by $C 6$ protects primary neurons against $A \beta 42$ species-induced neuronal apoptosis and tau dysregulation. (A) and (B) Immunoblot analysis and statistics of cleaved caspase-3, cleaved PARPI and DAPK1 function in WT primary neurons treated with $20 \mu \mathrm{M}$ A $\beta$ species alone or together with $2 \mu \mathrm{M}$ C6 for $24 \mathrm{~h}$. (C) and (D) Immunoblot analysis and statistics of total tau and tau phosphorylation at Thr231, Ser262 and Ser396 in WT primary neurons treated with A $\beta$ species alone or together with C6 for 24 h. $\beta$-actin was used as an internal control. Data are expressed as the mean \pm SD $(* p<0.05, * * p<0.01, * * * p<0.001, * * * * p<0.0001$, ns, not significant). 
A

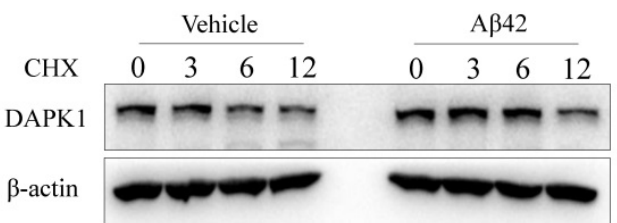

C

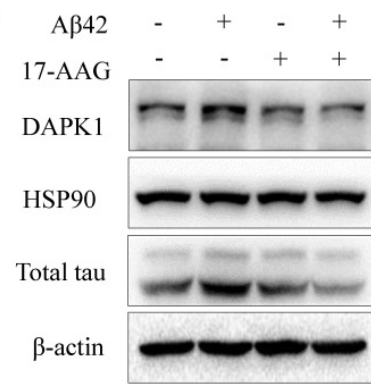

D

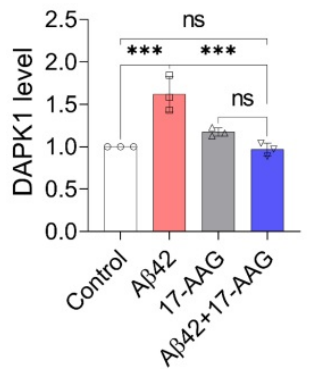

B

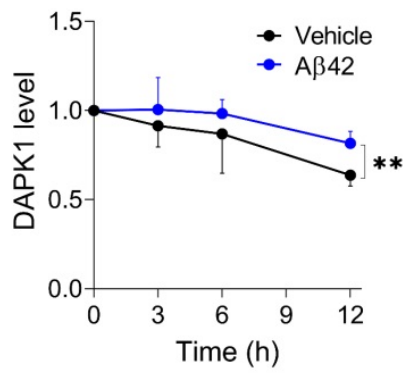

E

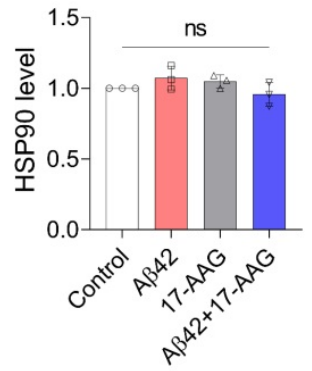

F

Figure 6. AB42 species stabilize DAPK1 protein by modulating HSP90 function. (A) and (B) $C H X$ assay to determine DAPK1 protein levels in WT primary neurons treated with vehicle or $20 \mu \mathrm{M} A \beta 42$ species for $0,3,6$ and $12 \mathrm{~h}$. The concentration of $\mathrm{CHX}$ was $10 \mu \mathrm{g} / \mathrm{ml}$. (C) Immunoblot analysis of WT primary neurons treated with $A \beta 42$ species alone or together with the HSP90 inhibitor 17-AAG $(200 \mathrm{nM})$ for $24 \mathrm{~h}$ to evaluate DAPK1, HSP90 and total tau levels. (D), (E) and (F) are the corresponding statistics. $\beta$-actin was used as an internal control. Data are expressed as the mean \pm SD $\left({ }^{*} p<0.05\right.$, $* * p<0.01$, $* * * p<0.001$, ns, not significant).

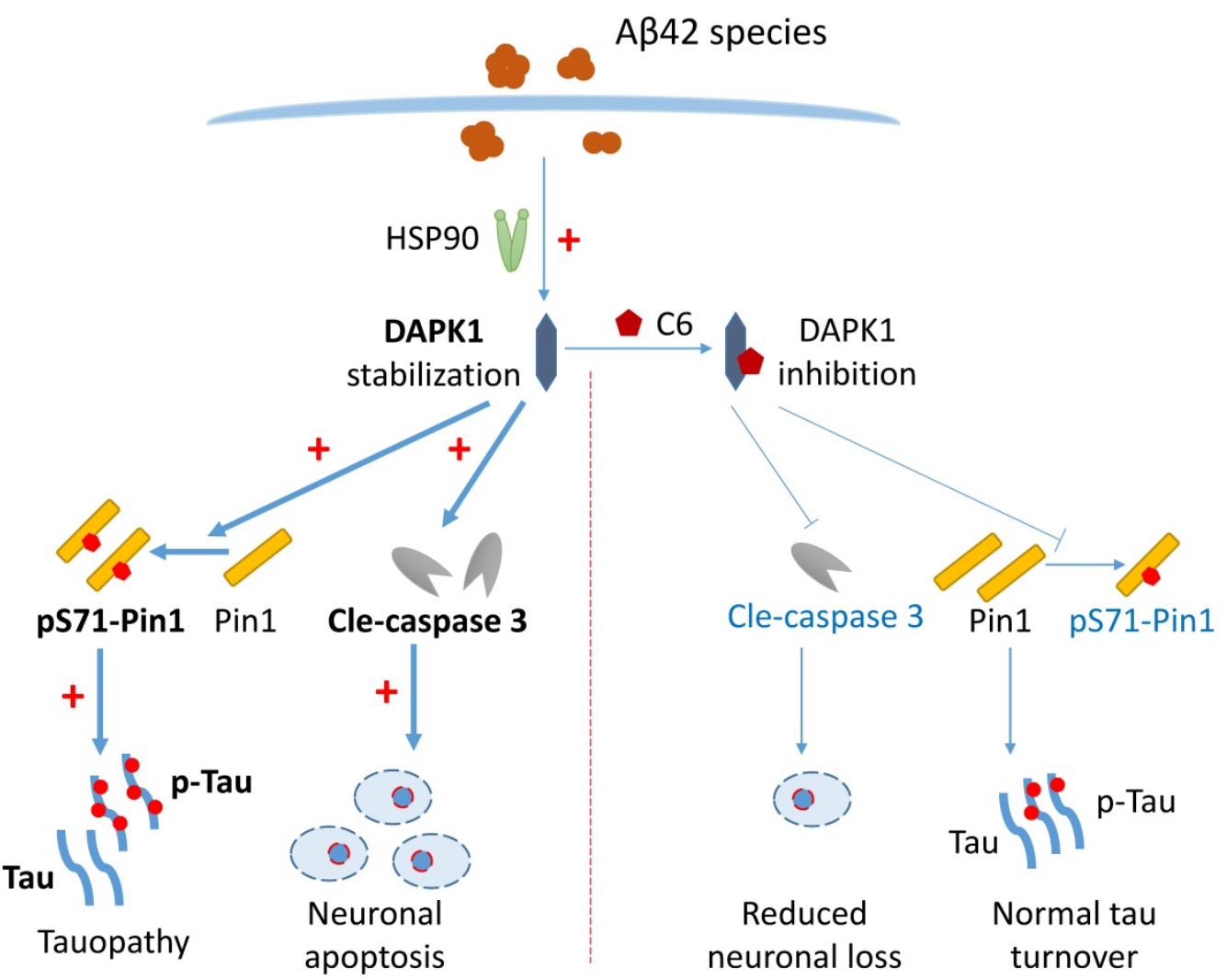

Figure 7. Schematic showing how A 342 species regulate DAPK1 and neuronal functions. The presence of $A \beta 42$ species leads to DAPK 1 stabilization and activation through the molecular chaperone HSP90. DAPK1 then inactivates Pin 1 by phosphorylating its Ser71 residue, resulting in tau accumulation and hyperphosphorylation in neurons. In addition, DAPK 1 activation also induces neuronal apoptosis by a caspase- 3 dependent mechanism. DAPK 1 inhibition by $\mathrm{C} 6$ protects neurons from A 3 -induced abnormal tau accumulation and mitigates neuronal loss. Upregulated proteins under AD conditions are shown in bold (left panel), and downregulated proteins caused by DAPK1 inhibition are depicted in blue (right panel). 


\section{Discussion}

Studies have revealed that $A \beta$ aggregation and tau hyperphosphorylation in the CNS may occur decades before significant cognitive dysfunction, whereas how the two pathological features of $\mathrm{AD}$ affect each other has not been fully resolved. In the present study, we used primary neurons as in vitro models to study effects of LMW and HMW A $\beta$ species on neuronal survival and tau phosphorylation. We discovered that $A \beta 42$ aggregates activate DAPK1 function through HSP90-mediated DAPK1 stabilization, which then leads to Pin1 inactivation in neurons by phosphorylating the Ser71 residue. Furthermore, Pin1 inhibition results in the accumulation of hyperphosphorylated tau and total tau proteins in primary neurons. In addition, the upregulation of DAPK1 also contributes to caspase-3 dependent neuronal apoptosis. Inhibiting DAPK1 could significantly prevent $A \beta$ aggregation-induced cell death and tau dysregulation. Our data illustrate that DAPK1 might be a key regulator of the crosstalk between $A \beta$ aggregation and tau dysfunction in $A D$.

Recent studies have emphasized that soluble $A \beta$ oligomers are the main contributor to neuronal dysfunction in the brain, causing extensive neurotoxic effects including neuroinflammation, oxidative stress and synaptic damage [6]. Despite the fact that the physiological concentration of $A \beta$ is well below the micromolar range [29], $A \beta$ oligomers have been shown to excessively accumulate in synaptic clefts and periplaque regions [37], and might reach micromolar concentrations [38]. The viability assay showed that both LMW and HMW A $\beta$ species are cytotoxic in a concentration-dependent manner, suggesting that our experimental conditions are still relevant to physiological conditions. Research has demonstrated that the neurotoxicity of $A \beta$ aggregates might be determined by the size and structural properties of these species. Small A $\beta$ species formed at the early stage of aggregation can efficiently disrupt cell membrane integrity, while large $A \beta$ species produced at a later stage of aggregation are prone to triggering neuroinflammatory responses [39]. In accordance with previous studies [26], LMW and HMW A $\beta 42$ species both contained small $A \beta$ oligomers below $15 \mathrm{kDa}$. HMW $\mathrm{A} \beta$ species are more heterogeneous than LMW $A \beta$ as they also have some larger $A \beta$ aggregates and fibrillar structures in solution. The partial overlap in the size distribution between LMW and HMW A $\beta$ species may explain similar outcomes of these two species in causing neuronal apoptosis and tau dysregulation. The analogous effects caused by LMW and HMW A $\beta$ species implicate that different $A \beta$ species share common pathological features in the CNS. However, this could also be attributed to a neuron-enriched condition used for assessing the effect of $A \beta$ species, as large $A \beta$ aggregates such as protofibrils tend to interact with glial cells rather than with neurons [39]. Nevertheless, we were not able to dissect the distinct functions of oligomeric and fibrillar $A \beta$ in regulating DAPK1 and tau under our experimental setup. Further studies are needed to characterize in detail how different $A \beta$ species influence neuronal functions.

The activation or upregulation of DAPK1 in the brain has been reported to contribute to synaptic loss, $\mathrm{A} \beta$ generation and tau hyperphosphorylation in $\mathrm{AD}$ $[12,40,41]$, while the molecular mechanism mediating DAPK1 upregulation in AD has not been revealed. A subsequent study found that A $\beta 25-35$ was able to activate DAPK1 through cathepsin B-mediated regulation [42], which seems to contradict previous research showing that cathepsin B deficiency increases the protein level of DAPK1 [43]. The regulation of DAPK1 function in cells has been recognized to be dominated by posttranslational mechanisms, such as proteasome- or lysosome-mediated protein degradation [43]. As an important executor of cellular proteostasis, HSP90 inhibition not only induces the degradation of DAPK1, but also significantly increases the level of DAPK1 phosphorylation at Ser308, synergistically leading to the suppression of DAPK1 function [10]. More importantly, HSP90 is upregulated and shows colocalization with $\mathrm{A} \beta$ in brains of $\mathrm{AD}$ patients [19]. We observed that both $A \beta 42$ preparations elevated DAPK1 protein levels in neurons without affecting its mRNA level, but through enhancing the protein stability of DAPK1. The application of the HSP90 inhibitor 17-AAG blocked DAPK1 elevation and tau accumulation induced by $A \beta$, indicating that HSP90 activation may act upstream of DAPK1 upregulation in this context. In light of the early onset of abnormal $\mathrm{A} \beta$ accumulation in the progression of $\mathrm{AD}$, we propose that DAPK1 upregulation induced by $A \beta$ aggregation might also be an early event in the disease course of AD. Since the activation of DAPK1 function could contribute to the amyloidogenic processing of APP and the hyperphosphorylation of tau [44], DAPK1 may serve as an important therapeutic target for the simultaneous control of both amyloidosis and tauopathy in AD.

Pin1 is a well-known phospho-specific prolyl isomerase that catalyzes the cis-trans conformational change in pSer/Thr-Pro bonds of target proteins. Tau phosphorylation at Thr231 (pT231-tau) is the only phosphorylation epitope in tau that can be recognized and isomerized by Pin1 [33]. Trans-ptau proteins 
possess normal microtubule binding affinity and can be dephosphorylated by protein phosphatases in neurons. They are also prone to undergo protein degradation and are therefore correlated with low neurodegenerative phenotypes [33]. In contrast, cis-ptau has completely opposite properties to trans-ptau and is extremely toxic to neurons [33]. Pin1 inactivation greatly reduces the degradation of cis-ptau, blocking the normal turnover of tau proteins in neurons as a consequence. Thus, the activity of Pin1 has a profound impact on intracellular tau homeostasis by balancing the conformational change of pT231-tau proteins. Consistent with our previous findings about DAPK1 and Pin1 [14], we found that the activation of DAPK1 by $A \beta 42$ species also led to Pin1 inactivation through Ser71 phosphorylation, which agrees with Sultana et al.'s finding that $\mathrm{AD}$ patients have a significant loss of Pin1 activity in the hippocampus compared with healthy controls [45]. Alternatively, $A \beta$ could modulate Pin1 function through promoting its dephosphorylation. Stallings et al. demonstrated that soluble $\mathrm{A} \beta 42$ species could increase calcineurin-mediated dephosphorylation of Pin1 at the Ser111 residue, thus downregulating its isomerase activity and eventually leading to dendritic spine loss in the brain [46]. However, the impact of A 342 species on Pin1 activity might be time-dependent, as Bulbarelli et al. reported that $A \beta 42$ oligomers may activate Pin1 by favoring the dephosphorylation of its Ser16 residue at the early stage of the treatment (e.g., 3-8 h). Pin1 function returned to the basal level after sustained $A \beta$ treatment (e.g., $24 \mathrm{~h}$ ). The transient activation of Pin1 by $A \beta$ could improve the dephosphorylation of pT231-tau, thereby reducing the overall phosphorylation of tau proteins in neurons [47]. This study also discovered that $A \beta$ fibrils had limited effects on Pin1 function, which is different from our results. We speculate that the difference might be related to the different properties of $A \beta$ species derived from the preparation protocols. It should also be noted that the function of GSK-3 $\beta$ and CDK5, the major tau-phosphorylating kinases in $\mathrm{AD}$, was not significantly altered by $A \beta$ species (Figure $S 4$ ), further supporting the crucial role of DAPK1 in the crosstalk between $A \beta$ and tau pathologies.

DAPK1 is widely accepted as an important regulator of neuronal cell death in a plethora of diseases including ischemic stroke, Parkinson's disease and AD [48]. For instance, DAPK1 has been shown to directly bind to extrasynaptic N-methyl-Daspartate receptors (NR2B) and phosphorylate its Ser1303 residue [49]. Upon phosphorylation by DAPK1, NR2B increases its conductance toward $\mathrm{Ca}^{2+}$, triggering intracellular $\mathrm{Ca}^{2+}$ overload that promotes neuronal cell death [49]. Furthermore, DAPK1 could also couple with p53 and phosphorylate its Ser23 residue. Phosphorylated p53 proteins can either translocate into the nucleus, where they can initiate proapoptotic gene transcription, or attach to the mitochondrial matrix to induce necrosis [50]. You et al. also revealed that DAPK1 is capable of binding to and phosphorylating $\mathrm{N}$-myc downstream-regulated gene 2 (NDRG2). DAPK1-induced NDRG2 phosphorylation at Ser350 promotes cell death through a caspase-3 dependent mechanism [51]. We found that DAPK1 KO or inhibition by $\mathrm{C} 6$ was able to alleviate neuronal apoptosis induced by both LMW and HMW A $\beta 42$ species by reducing caspase- 3 activation and PARP1 cleavage, suggesting that DAPK1 acts as a common upstream regulator of $A \beta 42$ aggregationinduced apoptosis. Pin1 is also a crucial player in modulating cell fate as it regulates the isomerization of a variety of phosphoproteins that are essential for the cell cycle, differentiation and cell survival [32]. The function of Pin1 in programmed cell death is defined by its cellular context. On the one hand, Pin1 may promote neuronal apoptosis by disrupting mitochondrial function or by activating the caspase- 3 pathway in developing neurons [52]. On the other hand, Pin1 may also exert antiapoptotic effects by stabilizing proteins of the Bcl-2 family in oligodendrocytes [52]. Although we did not have evidence to show that Pin1 inactivation by A $\beta$-induced DAPK1 upregulation played a direct role in neuronal apoptosis, the recovery of Pin1 function by DAPK1 inhibition correlated with the improvement in neuronal cell death. In contrast with Duan et al.'s report showing that DAPK1-mediated tau phosphorylation could counteract kinase-induced apoptosis in cell lines [53], the increased tau phosphorylation did not improve neuronal apoptosis in our study. We speculate that this is because $A \beta$ species are capable of causing cell death through different mechanisms [54]. This could also explain why inhibiting DAPK1 alone might be unable to completely reverse neuronal loss induced by $A \beta$. However, the significant reduction in apoptosis markers following DAPK1 inhibition implies that the DAPK1/Pin1 pathway could be a potential target for early intervention in $A \beta$ species-induced cellular damage.

In summary, our results show that $A \beta$ aggregates upregulate the protein level of DAPK1 through HSP90-mediated stabilization. The activation of DAPK1 subsequently results in a loss of function of Pin1 in primary neurons, leading to the abnormal accumulation of both total and hyperphosphorylated tau proteins (Figure 7). Additionally, DAPK1 upregulation by $A \beta$ aggregates also contributes to 
neuronal loss via a caspase-3 dependent mechanism, while its inhibition mitigates $A \beta$ aggregation-induced neuronal apoptosis and tau dysregulation (Figure 7). Our findings have uncovered a new mechanism through which different $A \beta$ aggregates evoke abnormal tau accumulation in neurons, and suggest that targeting the DAPK1/Pin1 pathway could be a useful strategy for the intervention in $A \beta$ aggregation-induced neuropathological changes throughout the progression of $\mathrm{AD}$.

\section{Abbreviations}

A $\beta$ : Amyloid- $\beta$; AD: Alzheimer's disease; DAPK1: Death-associated protein kinase 1; CSF: Cerebrospinal fluid; LMW: Low molecular weight; HMW: High molecular weight; GSK-3 $\beta$ : Glycogen synthase kinase-3 $\beta$; CDK5: Cyclin-dependent kinase 5; HSP90: Heat shock protein 90; PARP1: Poly-ADP-ribose polymerase-1; LOAD: Late onset Alzheimer's disease.

\section{Supplementary Material}

Supplementary figures and table. https://www.ijbs.com/v18p0693s1.pdf

\section{Acknowledgements}

This work received financial support from the National Natural Science Foundation of China (81970993 and 82001128), the Natural Science Foundation of Fujian Province (2019J01297 and 2021J01672) and Fujian Medical University (XRCZX2017019 and XRCZX2019039).

\section{Data availability}

All data generated or analyzed during this study are available from the corresponding author on reasonable request.

\section{Author Contributions}

THL and TZ conceived this project and wrote the manuscript. $\mathrm{YX}, \mathrm{TZ}$ and $\mathrm{LH}$ performed experiments and analyzed the data. DC, C-LG and LW provided advice to experiments. YM, GL, XS, YT, RL and MZ offered technical assistance. All authors discussed the results and contributed to the final manuscript.

\section{Competing Interests}

The authors have declared that no competing interest exists.

\section{References}

1. Scheltens P, De Strooper B, Kivipelto M, Holstege H, Chetelat G, Teunissen CE, et al. Alzheimer's disease. Lancet. 2021; 397: 1577-90.

2. Bateman RJ, Xiong C, Benzinger TL, Fagan AM, Goate A, Fox NC, et al. Clinical and biomarker changes in dominantly inherited Alzheimer's disease. N Engl J Med. 2012; 367: 795-804.
3. Larson ME, Lesne SE. Soluble Abeta oligomer production and toxicity. J Neurochem. 2012; 120 Suppl 1: 125-39.

4. Lesne SE, Sherman MA, Grant M, Kuskowski M, Schneider JA, Bennett DA, et al. Brain amyloid-beta oligomers in ageing and Alzheimer's disease. Brain. 2013; 136: 1383-98.

5. Yang T, Li S, Xu H, Walsh DM, Selkoe DJ. Large Soluble Oligomers of Amyloid beta-Protein from Alzheimer Brain Are Far Less Neuroactive Than the Smaller Oligomers to Which They Dissociate. J Neurosci. 2017; 37: 152-63.

6. Sengupta U, Nilson AN, Kayed R. The Role of Amyloid-beta Oligomers in Toxicity, Propagation, and Immunotherapy. EBioMedicine. 2016; 6: 42-9.

7. Zempel H, Thies E, Mandelkow E, Mandelkow EM. Abeta oligomers cause localized $\mathrm{Ca}(2+)$ elevation, missorting of endogenous Tau into dendrites, Tau phosphorylation, and destruction of microtubules and spines. J Neurosci. 2010; 30: 11938-50.

8. Jin M, Shepardson N, Yang T, Chen G, Walsh D, Selkoe DJ. Soluble amyloid beta-protein dimers isolated from Alzheimer cortex directly induce Tau hyperphosphorylation and neuritic degeneration. Proc Natl Acad Sci U S A. 2011; 108: 5819-24.

9. Amar F, Sherman MA, Rush T, Larson M, Boyle G, Chang L, et al. The amyloid-beta oligomer Abeta*56 induces specific alterations in neuronal signaling that lead to tau phosphorylation and aggregation. Sci Signal. 2017; 10: eaal2021.

10. Singh P, Ravanan P, Talwar P. Death Associated Protein Kinase 1 (DAPK1): A Regulator of Apoptosis and Autophagy. Front Mol Neurosci. 2016; 9: 1-11.

11. Bialik S, Kimchi A. The death-associated protein kinases: structure, function, and beyond. Annu Rev Biochem. 2006; 75: 189-210.

12. Kim BM, You MH, Chen CH, Lee S, Hong Y, Hong Y, et al. Death-associated protein kinase 1 has a critical role in aberrant tau protein regulation and function. Cell Death Dis. 2014; 5: e1237.

13. Xu LZ, Li BQ, Jia JP. DAPK1: a Novel Pathology and Treatment Target for Alzheimer's Disease. Mol Neurobiol. 2019; 56: 2838-44.

14. Lee $\mathrm{TH}$, Chen $\mathrm{CH}$, Suizu F, Huang P, Schiene-Fischer C, Daum S, et al. Death-associated protein kinase 1 phosphorylates Pin1 and inhibits its prolyl isomerase activity and cellular function. Mol Cell. 2011; 42: 147-59.

15. Wu PR, Tsai PI, Chen GC, Chou HJ, Huang YP, Chen YH, et al. DAPK activates MARK1/2 to regulate microtubule assembly, neuronal differentiation, and tau toxicity. Cell Death Differ. 2011; 18: 1507-20.

16. Pei L, Wang S, Jin H, Bi L, Wei N, Yan H, et al. A Novel Mechanism of Spine Damages in Stroke via DAPK1 and Tau. Cereb Cortex. 2015; 25: 4559-71.

17. Jin Y, Blue EK, Dixon S, Shao Z, Gallagher PJ. A death-associated protein kinase (DAPK)-interacting protein, DIP-1, is an E3 ubiquitin ligase that promotes tumor necrosis factor-induced apoptosis and regulates the cellular levels of DAPK. J Biol Chem. 2002; 277: 46980-6.

18. Zhang L, Nephew KP, Gallagher PJ. Regulation of death-associated protein kinase. Stabilization by HSP90 heterocomplexes. J Biol Chem. 2007; 282: 11795-804.

19. Kakimura J, Kitamura Y, Takata K, Umeki M, Suzuki S, Shibagaki K, et al. Microglial activation and amyloid-beta clearance induced by exogenous heat-shock proteins. FASEB J. 2002; 16: 601-3.

20. Chen Y, Wang B, Liu D, Li JJ, Xue Y, Sakata K, et al. Hsp90 chaperone inhibitor 17-AAG attenuates Abeta-induced synaptic toxicity and memory impairment. J Neurosci. 2014; 34: 2464-70.

21. Gozuacik D, Bialik S, Raveh T, Mitou G, Shohat G, Sabanay H, et al. DAP-kinase is a mediator of endoplasmic reticulum stress-induced caspase activation and autophagic cell death. Cell Death Differ. 2008; 15: 1875-86.

22. Jones SM, Novak AE, Elliott JP. Primary culture of cellular subtypes from postnatal mouse for in vitro studies of oxygen glucose deprivation. J Neurosci Methods. 2011; 199: 241-8.

23. Hilgenberg LG, Smith MA. Preparation of dissociated mouse cortical neuron cultures. J Vis Exp. 2007; 10: e562.

24. Stine WB, Jungbauer L, Yu C, LaDu MJ. Preparing synthetic Abeta in different aggregation states. Methods Mol Biol. 2011; 670: 13-32.

25. Stine WB, Jr., Dahlgren KN, Krafft GA, LaDu MJ. In vitro characterization of conditions for amyloid-beta peptide oligomerization and fibrillogenesis. J Biol Chem. 2003; 278: 11612-22.

26. Dahlgren KN, Manelli AM, Stine WB, Jr., Baker LK, Krafft GA, LaDu MJ. Oligomeric and fibrillar species of amyloid-beta peptides differentially affect neuronal viability. J Biol Chem. 2002; 277: 32046-53.

27. Glabe CG. Structural classification of toxic amyloid oligomers. J Biol Chem. 2008; 283: 29639-43.

28. Reifert J, Hartung-Cranston D, Feinstein SC. Amyloid beta-mediated cell death of cultured hippocampal neurons reveals extensive Tau fragmentation without increased full-length tau phosphorylation. J Biol Chem. 2011; 286: 20797-811.

29. Shin WS, Di J, Cao Q, Li B, Seidler PM, Murray KA, et al. Amyloid beta-protein oligomers promote the uptake of tau fibril seeds potentiating intracellular tau aggregation. Alzheimers Res Ther. 2019; 11: 86-98.

30. Gomes LA, Hipp SA, Rijal Upadhaya A, Balakrishnan K, Ospitalieri S, Koper MJ, et al. Abeta-induced acceleration of Alzheimer-related tau-pathology spreading and its association with prion protein. Acta Neuropathol. 2019; 138: 913-41.

31. Albayram O, Angeli P, Bernstein E, Baxley S, Gao Z, Lu KP, et al. Targeting Prion-like Cis Phosphorylated Tau Pathology in Neurodegenerative Diseases. J Alzheimers Dis Parkinsonism. 2018; 8: 443. 
32. Chen D, Wang L, Lee TH. Post-translational Modifications of the Peptidyl-Prolyl Isomerase Pin1. Front Cell Dev Biol. 2020; 8: 129.

33. Wang L, Zhou Y, Chen D, Lee TH. Peptidyl-Prolyl Cis/Trans Isomerase Pin1 and Alzheimer's Disease. Front Cell Dev Biol. 2020; 8: 355

34. Kim N, Chen D, Zhou XZ, Lee TH. Death-Associated Protein Kinase 1 Phosphorylation in Neuronal Cell Death and Neurodegenerative Disease. Int J Mol Sci. 2019; 20: 3131.

35. Okamoto M, Takayama K, Shimizu T, Ishida K, Takahashi O, Furuya T. Identification of death-associated protein kinases inhibitors using structure-based virtual screening. J Med Chem. 2009; 52: 7323-7.

36. Evans CG, Wisen S, Gestwicki JE. Heat shock proteins 70 and 90 inhibit early stages of amyloid beta-(1-42) aggregation in vitro. J Biol Chem. 2006; 281: 33182-91.

37. Bush AI, Tanzi RE. Therapeutics for Alzheimer's disease based on the metal hypothesis. Neurotherapeutics. 2008; 5: 421-32.

38. Zhang F, Gannon M, Chen Y, Yan S, Zhang S, Feng W, et al. beta-amyloid redirects norepinephrine signaling to activate the pathogenic GSK3beta/tau cascade. Sci Transl Med. 2020; 12: eaay6931.

39. De S, Whiten DR, Ruggeri FS, Hughes C, Rodrigues M, Sideris DI, et al. Soluble aggregates present in cerebrospinal fluid change in size and mechanism of toxicity during Alzheimer's disease progression. Acta Neuropathol Commun. 2019; 7: 120-32.

40. Shu S, Zhu H, Tang N, Chen W, Li X, Li H, et al. Selective Degeneration of Entorhinal-CA1 Synapses in Alzheimer's Disease via Activation of DAPK1. J Neurosci. 2016; 36: 10843-52.

41. Kim BM, You MH, Chen $\mathrm{CH}$, Suh J, Tanzi RE, Ho Lee T. Inhibition of death-associated protein kinase 1 attenuates the phosphorylation and amyloidogenic processing of amyloid precursor protein. Hum Mol Genet. 2016; 25: 2498-513

42. Song L, Pei L, Hu L, Pan S, Xiong W, Liu M, et al. Death-associated protein kinase 1 mediates interleukin-1beta production through regulating inlfammasome activation in Bv2 microglial cells and mice. Sci Rep. 2018; 8: 9930-40.

43. Lin $\mathrm{Y}$, Stevens $\mathrm{C}$, Hupp T. Identification of a dominant negative functional domain on DAPK-1 that degrades DAPK-1 protein and stimulates TNFR-1-mediated apoptosis. J Biol Chem. 2007; 282: 16792-802.

44. Chen D, Zhou XZ, Lee TH. Death-Associated Protein Kinase 1 as a Promising Drug Target in Cancer and Alzheimer's Disease. Recent Pat Anticancer Drug Discov. 2019; 14: 144-57.

45. Sultana R, Boyd-Kimball D, Poon HF, Cai J, Pierce WM, Klein JB, et al. Oxidative modification and down-regulation of Pin1 in Alzheimer's disease hippocampus: A redox proteomics analysis. Neurobiol Aging. 2006; 27: 918-25.

46. Stallings NR, O'Neal MA, Hu J, Kavalali ET, Bezprozvanny I, Malter JS. Pin1 mediates Abeta42-induced dendritic spine loss. Sci Signal. 2018; 11: eaap8734.

47. Bulbarelli A, Lonati E, Cazzaniga E, Gregori M, Masserini M. Pin1 affects Tau phosphorylation in response to Abeta oligomers. Mol Cell Neurosci. 2009; 42: 75-80.

48. Sulaiman Alsaadi M. Role of DAPK1 in neuronal cell death, survival and diseases in the nervous system. Int J Dev Neurosci. 2019; 74: 11-7.

49. Tu W, Xu X, Peng L, Zhong X, Zhang W, Soundarapandian MM, et al. DAPK1 interaction with NMDA receptor NR2B subunits mediates brain damage in stroke. Cell. 2010; 140: 222-34.

50. Wang S, Shi X, Li H, Pang P, Pei L, Shen H, et al. DAPK1 Signaling Pathways in Stroke: from Mechanisms to Therapies. Mol Neurobiol. 2017; 54: 4716-22.

51. You MH, Kim BM, Chen CH, Begley MJ, Cantley LC, Lee TH. Death-associated protein kinase 1 phosphorylates NDRG2 and induces neuronal cell death. Cell Death Differ. 2017; 24: 238-50.

52. Fagiani F, Govoni S, Racchi M, Lanni C. The Peptidyl-prolyl Isomerase Pin1 in Neuronal Signaling: from Neurodevelopment to Neurodegeneration. Mol Neurobiol. 2021; 58: 1062-73.

53. Duan DX, Chai GS, Ni ZF, Hu Y, Luo Y, Cheng XS, et al. Phosphorylation of tau by death-associated protein kinase 1 antagonizes the kinase-induced cell apoptosis. J Alzheimers Dis. 2013; 37: 795-808.

54. Chi H, Chang HY, Sang TK. Neuronal Cell Death Mechanisms in Major Neurodegenerative Diseases. Int J Mol Sci. 2018; 19: 3082. 